\title{
Omega-3 fatty acids in psychological disorders
}

\author{
D Ariyasinghe, SS Williams
}

\section{A randomised, placebo controlled trial of omega-3 fatty acids in the treatment of young children with autism (1)}

Omega-3 fatty acids have been used to treat various symptoms in autism spectrum disorders (ASD). Studies done previously have given mixed results. In the current study, the authors examined the effectiveness of omega 3 fatty acids in the treatment of ASD using a randomised, double blind placebo controlled design.

A total of 38 children including 28 males and 10 females with ASD, aged between 2 to 5 years were randomised after taking informed consent from their parents. The study period was 6 months. The diagnosis of ASD was established using DSM IV(TR) criteria. The diagnosis was supported by the Autism Diagnostic Observation Schedule and the Autism Diagnostic Interview - Revised.

Outcome measures were autism symptom severity and externalising behaviors. Autism symptom severity was measured by the composite autism score of the Pervasive Developmental Disorder - Behavioral Inventory (PDDBI) and externalising behaviours were measured using the Behaviour Assessment System for Children, Second Edition (BASC-2). Both investigators and the participants were blinded to the medication given.

The starting dose of EPA (eicosapentaenoic acid) + DHA (docosahexaenoic acid) was $0.75 \mathrm{~g}$ and if it was tolerated it was doubled after 2 weeks. The participants were seen every 2 weeks during the first 4 weeks and every 4 weeks thereafter.

The statistical model used was mixed model regression analysis. For the PDDBI and BASC-2, the change from baseline at 12 weeks and 24 weeks was analysed, with baseline scores and sex in the model as potential confounders.

Statistical analysis did not reveal any significant difference between groups on PDDBI autism composite scores at weeks 0 and 24. But there was a significant change by week 24 on BASC-2 externalizing problem score. Participants randomised to placebo showing a mild improvement where as the treatment group demonstrated worsening scores at week 24.
Authors concluded that there is no data to support the hypothesis that supplementation with omega-3 fatty acids is beneficial in preschoolers with ASD. Worsening of externalizing behaviours observed in the treatment group may have been due to increased gastrointestinal side effects. The authors acknowledge that small sample size is a limitation of the study.

\section{Omega-3 fatty acid supplementation for the treatment of children with attention deficit hyperactivity disorder symptomatology: Systematic review and meta-analysis (2)}

The use of omega 3 fatty acids in the treatment of attention deficit hyperactivity disorder (ADHD) is widespread. The aim of the study was to conduct a meta-analysis to determine the efficacy of omega-3 fatty acid supplementation in ADHD and to use meta-regression to examine the difference in treatment efficacy of different omega-3 fatty acid compositions.

The reviewers searched PubMed and the cinicaltrials. gov website using the terms 'attention deficit disorder with hyperactivity, fatty acids, unsaturated, omega- 3 and omega-6'. Only the studies which had a randomised placebo-controlled design to examine the efficacy of omega-3 fatty acid supplementation in children with ADHD and which used a validated rating scale to measure ADHD severity during the trial were included. If the children had been started on psychoactive substance at the same time they were excluded.

The standard mean difference (SMD) was chosen as the summary statistic for meta-analysis and was calculated by pooling the standardised mean improvement of each study using, Rev Man 5. Meta-regression was performed with SPSS 19.0, using linear regression.

Ten eligible trials with 11 appropriate treatment arms and a total of 699 participants were included in the analysis. Trial durations ranged from 4 weeks to 4 months.

A small but significant effect of omega-3 fatty acid supplementation for ADHD was found. This was seen for both inattentive and hyperactive symptoms. Higher doses of eicosapentaenoic acid (EPA) within omega-3 fatty acids supplements were significantly associated with increased efficacy in treating ADHD symptoms, 
although this effect was not observed with regard to docosahexaenoic acid or $\alpha$-linolenic acid.

The authors acknowledged several limitations of the trials included in the review, such as poor randomisation and not accounting for drop-outs.

The authors conclude that the currently available evidence is not sufficient to recommend omega- 3 fatty acid supplementation as the sole treatment in children with significant ADHD symptoms. However, given evidence of modest efficacy, particularly with higher doses of EPA, it can be used for augmentation of traditional pharmacotherapy or for those families who are reluctant to use psychopharmacological agents.

A randomised, controlled, crossover trial of fish oil treatment for impulsive aggression in children and adolescents with disruptive behavior disorders (3)

The primary aim of this study was to assess the efficacy of omega-3 fatty acids in the treatment of impulsive aggression in children and adolescents diagnosed with oppositional defiant disorder or conduct disorder. The diagnosis was made by structured interview: Kiddie Schedule for Affective Disorders and Schizophrenia for School-Aged Children - Present and Lifetime Version [K-SADS-PL)

In addition, general emotional and behavioural functioning, symptoms of hyperactivity, family functioning, and cognitive functioning were also assessed.

Outcome measures were assessed using the Children's Aggression Scale - Parent Version (CAS parent), the Modified Overt Aggression Scale (MOAS), Strengths and Difficulties Questionnaire (SDQ), Family Assessment Device General Functioning Scale (FAD-GFS), ADHD Rating Scale, Stop Signal Task, Trail Making Task and Eriksen Flanker Task.

The trial was a prospective, randomised, placebocontrolled, crossover trial comparing fish oils with placebo, conducted over 12 weeks. Children aged between 6-17 years and who had exhibited impulsive aggressive behaviours persisting for at least 6 months, and who had received at least one unsuccessful attempt at behavioral intervention for aggression, were included in the study. Children diagnosed with other neurodevelopmental disorders or other major psychiatric illnesses were excluded from the trial.

The participants were randomised to receive either 6 weeks of fish oil (4 g daily, containing $400 \mathrm{mg}$ eicosapentaenoic acid and $2000 \mathrm{mg}$ dicocosahexaenoic acid) followed by 6 weeks of placebo or 6 weeks of placebo, followed by 6 weeks of fish oil. Twenty-one children were randomised; 12 in the group starting with fish oil and 9 in the group starting with placebo. Clinical assessments were conducted every 2 weeks. Research assessments were conducted at baseline, 6 weeks, and 12 weeks. Treatment outcomes were analysed using mixed-effects modeling.

Results showed no clinically significant benefit of omega 3 fatty acids in children with aggressive behaviour, and on the contrary, it worsened the aggression. Benefits were not observed with regard to cognitive functioning. The authors conclude that the available evidence does not indicate the use of omega 3 fatty acids in the treatment of aggressive behaviours in children.

\section{Omega-3 fatty acids for the treatment of dementia (4)}

Omega-3 polyunsaturated fatty acids (omega-3 PUFAs) from fish and plant sources are commonly considered as a promising non-medical alternative to improve brain functions and slow down the progression of dementia. This assumption is mostly based on findings of preclinical studies and epidemiological research.

This Cochrane review assesses the efficacy and safety of omega-3 polyunsaturated fatty acid (PUFA) supplementation for the treatment of people with dementia.

They searched the Specialized Register of the Cochrane Dementia and Cognitive Improvement Group (ALOIS), MEDLINE, EMBASE, PsycINFO, CINAHL, Clinical Trials.gov and the World Health Organization (WHO) portal/ICTRP on 10 December 2015. They also contacted manufacturers of omega-3 supplements and scanned reference lists of landmark papers.

They included randomised controlled trials in which omega-3 PUFA in the form of supplements or enriched diets were administered to people with Alzheimer's disease (AD), vascular dementia (VaD), dementia with Lewy bodies (DLB), Parkinson's disease dementia (PDD) or frontotemporal dementia (FTD).

The primary outcome measures of interest were changes in global and specific cognitive functions, functional performance, dementia severity and adverse effects. Two review authors independently selected studies, extracted data and assessed the quality of trials according to the Cochrane Handbook for Systematic Reviews of Interventions. They rated the quality of the evidence using the GRADE approach.

They included three comparable randomised, placebocontrolled trials investigating omega-3 PUFA supplements in 632 participants with mild to moderate AD over six, 12 and 18 months. They found no studies investi- 
gating other types of dementia. All trials were of high methodological quality.

The authors conclude that there is no convincing evidence for the efficacy of omega-3 PUFA supplements in the treatment of mild to moderate AD. This result was consistent for all outcomes relevant for people with dementia. Adverse effects of omega-3 PUFAs seemed to be low, but based on the evidence in this review, the authors do not make a final statement on tolerability.

\section{A randomised, controlled trial of omega-3 fatty acids plus an antioxidant for relapse prevention after antipsychotic disconti- nuation in first-episode schizophrenia (5)}

This study investigated whether a combination of omega-3 polyunsaturated fatty acids ( $\omega$-3 PUFAs) and a metabolic antioxidant, alpha-lipoic acid ( $\alpha$-LA), is effective in preventing relapse after antipsychotic discontinuation, in subjects who were successfully treated for 2-3 years after a first-episode of schizophrenia, schizoaffective or schizophreniform disorder.

In this randomized, double-blind, placebo controlled study, antipsychotic treatment was tapered and discontinued and participants received either $\omega$-3 PUFAs (eicosapentaenoic acid 2g/day and docosahexaenoic acid $1 \mathrm{~g} /$ day $)+\alpha$-LA $300 \mathrm{mg} /$ day or placebo. Subjects were followed up for two years, or until relapse.

Recruitment was terminated prematurely due to the high relapse rates in both groups as well as the severity of some of the relapse episodes. The cumulative 2-year relapse rate was $19 / 21(90 \%)$ among participants randomized to $\omega$-3 PUFAs $+\alpha-L A$ and 9/12 (75\%) among those on placebo. Among those on $\omega-3$ PUFAs $+\alpha-L A$ only one participant (5\%) completed two years without relapse $(\mathrm{p}=0.6)$ and none on placebo completed the two years without relapse. Mean times to relapse were $39.8 \pm$ 25.4 and $38.3 \pm 26.6$ weeks for the $\omega$-3 PUFAs $+\alpha$-LA and placebo groups, respectively $(\mathrm{p}=0.9)$. There were no significant differences between the groups in relapse symptom severity.

The authors conclude that there is no evidence to indicate that $\omega$-3 PUFAs $+\alpha$-LA is a suitable alternative to maintenance antipsychotic treatment in relapse prevention. Although the small study size is a limitation, evidence from this study suggests that antipsychotic discontinuation after a single episode of schizophrenia carries a very high risk of relapse.

\section{Declaration of interest}

None declared

D Ariyasinghe, Department of Psychiatry, Faculty of Medicine, University Peradeniya

SS Williams, Department of Psychiatry, Faculty of Medicine, University Kelaniya

Corresponding author: D Ariyasinghe

Email: dewasmika.ariyasinghe@gmail.com

\section{References}

1. Mankad D, Dupuis A, Smile S, Roberts W, Brian J, Lui T, et al. A randomized, placebo controlled trial of omega-3 fatty acids in the treatment of young children with autism. Mol Autism 2015; 6: 18.

2. Bloch MH, Qawasmi A. Omega-3 fatty acid supplementation for the treatment of children with attentiondeficit/hyperactivity disorder symptomatology: systematic review and meta-analysis. J Am Acad Child Adolesc Psychiatry 2011; 50(10): 991-1000.

3. Dean AJ, Bor W, Adam K, Bowling FG, Bellgrove MA. A randomized, controlled, crossover Trial of fish oil treatment for impulsive aggression in children and adolescents with disruptive behavior disorders. Journal Child Adolesc Psychopharmacol 2014; 24(3): 140-8.

4. Burckhardt M, Herke M, Wustmann T, Watzke S, Langer G, Fink A. Omega-3 fatty acids for the treatment of dementia. Cochrane Database Syst Rev. 2016; 4: CD009002.

5. Emsley R, Chiliza B, Asmal L, du Plessis S, Phahladira L, van, Niekerk E, van Rensburg SJ, Harvey BH. A randomized, controlled trial of omega-3 fatty acids plus an antioxidant for relapse prevention after antipsychotic discontinuation in first-episode schizophrenia. Schizophr Res 2014; 158(1-3): 230-5. 